\title{
Bei jungen Patienten lauern juristische Fallstricke
}

\author{
Wenn Ärzte Jugendliche behandeln, kann das für sie schnell zivil-, berufs- und strafrecht- \\ liche Folgen haben, auch wenn die Therapie erfolgreich war. Zu beachten gilt nicht nur \\ die Schweigepflicht.
}

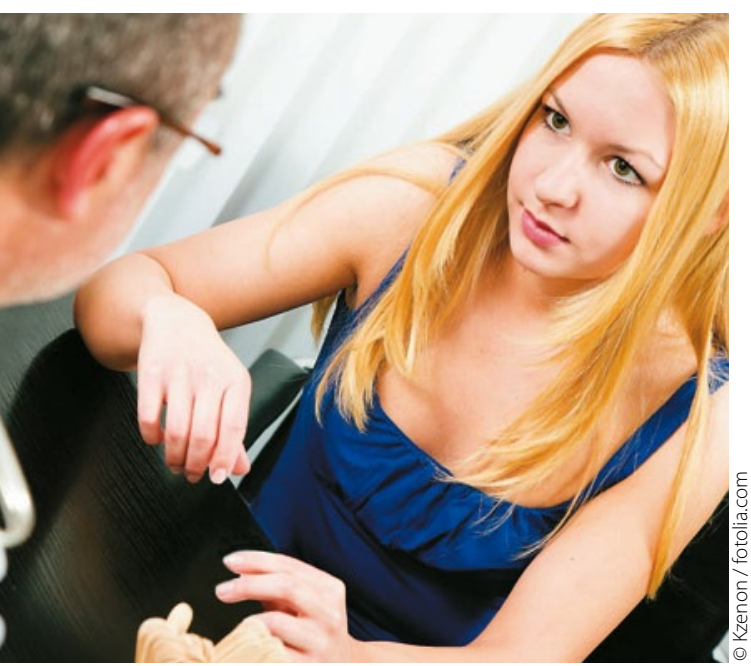

Darf der Arzt Minderjährige ohne Einwilligung der Eltern behandeln?

- Ob der Wunsch nach der Pille, eine ungewollte Schwangerschaft oder eine banale Erkältung: Jeden Tag suchen Jugendliche ohne Elternbegleitung den Arzt auf. Doch dürfen Ärzte Minderjährige ohne Einwilligung der Eltern überhaupt behandeln? „Die gesetzlichen Regelungen sind nicht immer eindeutig“, warnte Martin Stellpflug, Fachanwalt für Medizinrecht, auf dem Symposium „Medizin und Recht in Kindheit und Alter" in Berlin.

Damit der Eingriff in die körperliche Integrität eines Patienten gerechtfertigt ist, muss dieser in die Behandlung einwilligen. Sonst gilt auch eine indizierte Behandlung generell als Körperverletzung und ist, auch wenn sie fehlerfrei verläuft, strafbar. Bei jüngeren Kindern ist die Rechtslage eindeutig: Hier müssen die Sorgeberechtigten, üblicherweise die Eltern, nach einer Aufklärung mit der Behandlung einverstanden sein.

\section{Ist die Einsichtsfähigkeit gegeben?}

Schwieriger wird es bei Jugendlichen: „Denn bei der Einwilligung kommt es nicht auf die Geschäftsfähigkeit an“, erklärte Stellpflug. Entscheidend sei die natürliche Einsichts- und Urteilsfähigkeit. „Es ist davon auszugehen, dass die Einsichtsfähigkeit regelmäßig etwa ab dem 14. Lebensjahr vorliegt“, sagte der Fachanwalt. Dessen ungeachtet sollte der Arzt in der jeweils konkreten Situation sorgfältig prüfen, ob der Jugendliche einwilligungsfähig ist.

In der Rechtsprechung ist es laut Stellpflug allerdings umstritten, ob ein einwilligungsfähiger Patient automatisch auch alleine entscheidungsbefugt ist, das heißt, ob die Eltern informiert werden müssen oder nicht. Während das Bundesverfassungsgericht (BVerfG) davon ausgeht, dass ein urteilsfähiger Minderjähriger seine Persönlichkeitsrechte eigenständig ausüben kann, bestimmte der Bundesgerichtshof (BGH) unter anderem in einem Urteil aus dem Jahre 2006, dass bei der Aufklärung vor schwerwiegenden Eingriffen auch bei einsichtsfähigen Minderjährigen stets beide Elternteile aufgeklärt werden müssen.

„Das Patientenrechtegesetz scheint sich zugunsten des Bundesverfassungsgerichts entschieden zu haben“, lautet die Einschätzung des Fachanwalts. $\mathrm{Ob}$ die Rechtsprechung dieser Einschätzung folgt, bleibe allerdings abzuwarten. Auch viele Ärzte sind der Ansicht, Minderjährige sollten selbst entscheiden können, ob sie in eine Behandlung einwilligen oder nicht, wenn sie die erforderliche Reife besitzen.
„Es ist einigermaßen grotesk, dass ein 17-Jähriger zwar ein Auto fahren darf und ernsthaft erwogen wird, 16-Jährige mit aktivem Wahlrecht auszustatten, Ärzte aber für die Einwilligung zur Behandlung im Normalfall bis zum 18. Lebensjahr die Zustimmung der Eltern benötigen“, kritisierte Prof. Harald Mau, der bis zu seiner Emeritierung fast 20 Jahre lang die Kinderchirurgische Klinik der Berliner Charité leitete.

\section{Schweigepflicht auch gegenüber den Eltern?}

Wie sieht es also mit der Schweigepflicht gegenüber Eltern aus? „Hält ein Arzt seinen Patienten für einwilligungsfähig, kann der Minderjährige aber auf der Schweigepflicht bestehen", ist Anwalt Stellpflug überzeugt. Gegen den Willen eines voll einsichts- und urteilsfähigen Minderjährigen darf der Arzt die Eltern im Regelfall nicht informieren. Dennoch sollten Ärzte, so seine Empfehlung, versuchen, den Jugendlichen davon zu überzeugen, die Eltern vor allem bei schwerwiegenderen Eingriffen in die Entscheidung einzubeziehen.

In jedem Fall sollten Ärzte den gesamten Behandlungsablauf exakt dokumentieren. Eine detaillierte Dokumentation kann vor späteren rechtlichen Unklarheiten schützen und schafft für alle Beteiligten die Sicherheit, die einzelnen Schritte auch im Nachhinein verfolgen zu können. Ärzte sollten daher nicht nur festhalten, welche Entscheidungen sie getroffen haben, sondern auch, warum sie sich für diesen Weg entschieden haben.

EUGENIE WULFERT 